\title{
36. Développement et innovation au niveau des politiques municipales des personnes âgées
}

C S Springer-Verlag France 2010

\author{
36.2 \\ Une étude objective des territoires de vie \\ de personnes âgées : une analyse de la mobilité \\ au moyen de traceurs GPS \\ P.-M. Chapon ${ }^{1}$, F. Renard ${ }^{2}$, S. Habi ${ }^{3}$, J. Gueslot ${ }^{3}$, M. Dautan ${ }^{4}$, \\ P. Mallea ${ }^{5}$, P. Robert ${ }^{6}$, O. Guerin ${ }^{7}$ \\ ${ }^{1} I C A D E$ \\ ${ }^{2} U M R 5600$ \\ ${ }^{3}$ Université Paris 12, Paris, France \\ ${ }^{4}$ Faculté de médecine \\ ${ }^{5} \mathrm{DRCI}, \mathrm{CHU}$ de Nice, France \\ ${ }^{6} \mathrm{CMRR}, \mathrm{CHU}$ de Nice, France \\ ${ }^{7}$ Service de gériatrie CHU de Nice, Nice, France
}

Le vieillissement de la population constitue un défi pour les urbanistes et les planificateurs mais peu d'études se penchent sur l'étude des déplacements quotidiens des personnes âgées.

Objectifs : Ce présent article à vocation à présenter les premiers résultats d'une étude collaborative entre médecins et géographes étudiant les territoires de vie d'un échantillon de personnes âgés vivant dans différents territoires au sein de la ville de Nice.

Les volontaires équipées de traceurs GPS ont également passé une série de dépistages effectués par l'équipe médicale du centre hospitalier universitaire (CHU) de Nice. D'autres facteurs comme le type d'habitat ou encore la Catégorie Socioprofessionnelle (CSP) d'origine ont été pris en compte. L'utilisation de GPS permet de révéler de manière objective l'ensemble des déplacements qui peuvent ainsi être comparés aux données médicales de chaque individu recueillies par les médecins.

Les premiers résultats laissent apparaître que la qualité de l'environnement géographique impacte peu sur le degré de l'isolement des résidents. Les deux principaux facteurs mis en évidence sont la présence de troubles cognitifs et la dépression.

L'analyse des habitudes de vie permet de repenser les villes en fonction des profils établis de personnes âgées. Ainsi, on peut dégager des optimums d'implantation pour des logements à l'architecture adaptée, résidences services ou encore les EHPAD. Ces éléments intéressent tout particulièrement les urbanistes mais également les promoteurs immobiliers qui peuvent proposer une offre d'hébergement qui correspond à la fois à un profil établi de population à un territoire donné. En outre, la connaissance des territoires de vie des personnes auditées donne un éclairage nouveau sur les corrélations entre la qualité de l'environnement géographique et des problématiques telles que la dépression ou la solitude. Le rôle des qualités intrinsèques d'un territoire peut ainsi être repéré et corrigé le cas échéant dans une démarche préventive.

\section{3}

Villes amies des aînés au Québec :

la rencontre de la société civile, de l'administration municipale et du politique

S. Garon, M. Beaulieu

Université de Sherbrooke, centre de recherche sur le vieillissement du CSSS-IUGS, Sherbrooke, Québec, Canada

Le programme Villes amies des aînés au Québec, lancé en 2008 (pour 5 ans, donc jusqu'en 2013), sous forme de sept projets pilotes et implanté par la suite via le programme Municipalité amie des aînés sur l'ensemble du territoire québécois en 2010, propose, dans un contexte de vieillissement accéléré de la population, une structure de concertation entre divers acteurs du monde municipal. Dans une visée de vieillissement actif qui, selon l'OMS (2002) « est le processus consistant à optimiser les possibilités de bonne santé, de participation et de sécurité afin d'accroître la qualité de vie pendant la vieillesse » (p. 12), chaque municipalité est appelée à introduire des changements tant au plan social, culturel, structurel, etc. Pour favoriser ces travaux, diverses instances furent mises en place dont, dans chaque municipalité, la création d'un comité de pilotage où les acteurs ne partagent pas toujours un long parcours de collaboration en commun. De cultures organisationnelles distinctes, ayant des modalités de reconnaissance et légitimation différentes sinon divergentes, ces groupes d'acteurs — dont des représentants d'associations d'aînés —, que l'on peut réunir selon leurs fonctions de la manière suivante soit, la société civile, l'administration municipale et le politique, sont pourtant interpellés pour repenser l'espace municipal en tenant compte des besoin des aînés.

Dans le cadre de notre exposé, en plus de présenter les innovations récentes qui résultent de Villes amies des aînés au Québec depuis deux ans, nous ferons état de résultats préliminaires de l'évaluation d'implantation. Différents cas de figures, que peut prendre cette collaboration entre la société civile, l'administration municipale et le politique seront discutés afin d'en souligner les forces et les faiblesses. 